\title{
MOSSBAUER AND MAGNETIZATION STUDIES OF AMORPHOUS NAFeB COMPOSITIONALLY MODULATED THIN FILMS
}

B. Martinez $z^{+}$M. A. Moreu, A. Ruiz ${ }^{++}$

A. Labarta, X. Obradors, F. Briones ${ }^{++}$,

J. Tejada.

Fac. Fisica, Diagonal 647, 08028 Barcelona

+ I. C. M. A. B., C. S. I. C., Martr i Franqués

$s / n, 08028$ Barcelona

++ C. N. M. - C. S. I. C. , Serrano 144, Madrid

$$
\text { SPAIN }
$$

\section{ABSTRACT}

In this work we have studied several $\mathrm{NdFeB}$ compositionally modulated thin films by using both conversion electron Mössbauer spectra and SQUID magnetometry. Both the

hyperfine fields and the easy magnetization magnitude are not correlated with the modulation characteristic length $(\lambda)$ while the magnetization perpendicular to the thin film plane decreases as $\lambda$ increases.

\section{INTRODUCTION}

In the research of thin solid films and multilayered samples cortaining iron, the use of both the electron conversion Mössbauer spectroscopy and SQUID magnetometry are of enormous importance in order to detect the interfacial effects and the change in the magnetic properties with varying the composition or (and) the modulation length $1-3)$.

\section{EXPERIMENTAL RESULTS AND DISCUSSION}

The NdFeB compositionally modilated thin films were prepared in a special triode sputtering system onto glass substrates held at room temperature.

Single layers of an amorphous alloy of composition $\mathrm{Nd}_{26} \mathrm{~B}_{6} \mathrm{Fe}_{68}$ with different thicknesses are separated by amorphous single layers of $\mathrm{B}_{8} \mathrm{Fe} 92$. The total thickness of the thin films were determined by using a Tolansky interferometer $(1500 \AA)$

By means of $X-r a y$ diffraction we verified the amorphous character of the samples and using low angle $X$-ray scattering we tested the modulating structure of the samples.

With the aim to compare our data on thin films with those characteristics of bulk samples we have prepared a sample of composition $\mathrm{Nd}_{12} \mathrm{~B}_{8} \mathrm{Fe} 80$ having a thickness of about 2000 \& (This sample will be denoted with the number 6 ).
In table $I$ we summarize the mean characteristics of the obtained thin films.

\begin{tabular}{|c|c|c|}
\hline \multicolumn{3}{|c|}{$\begin{array}{l}\text { TABLE I: Thickness and modulation length } \\
\text { of the samples }\end{array}$} \\
\hline SAMPLE & $\begin{array}{l}\text { Nd26 }{ }_{6} \mathrm{Fe}_{28} \\
\text { SINGLE LAYER } \\
\text { THICKNESS ( })\end{array}$ & $\begin{array}{l}\text { MODULATION } \\
\text { LENGTH } \\
\text { (\&) }\end{array}$ \\
\hline $\begin{array}{l}1 \\
2 \\
3 \\
4 \\
5\end{array}$ & $\begin{array}{r}2.3 \\
4.6 \\
9.1 \\
18.2 \\
36.5\end{array}$ & $\begin{array}{r}3.4 \\
6.9 \\
13.7 \\
27.3 \\
54.7\end{array}$ \\
\hline
\end{tabular}

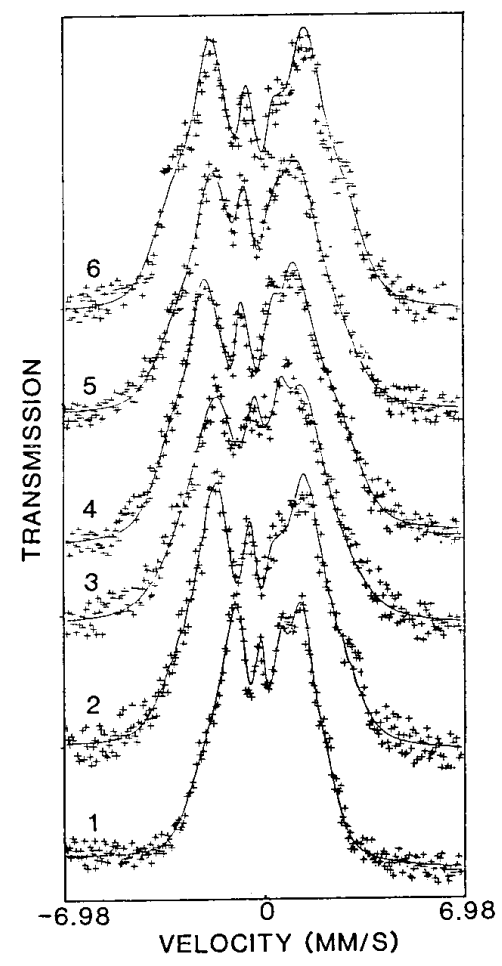

1GURE 1: Spectra of the NdFeB samples for different $\lambda$ values. 
The electron conversion Mössbauer studies were carried out by using an acetone gas detector. The spectra were recorded at room temperature being the gamma rays perpendicular to the substrate plane. The magnetization measurements were recorded by using a SHE SQUID magnetometer in applied magnetic fields up to $5.5 \mathrm{~T}$ and in the temperature range between 1.8 and $300 \mathrm{~K}$.

The Mössbauer spectra consist of six broad partially overlapped lines (see fig. 1) wich have been fitted by assuming:

a) the existence of both a distribution function, $P(H)$, for the magnetic field and a pure quadrupole doublet wich takes into account the existence of non magnetic $F e$ ions 5 ).

b) We have taken $P(H)$ as a binomial distribution being fixed the number of fields to be considered $4-5$ ).

c) The intensity ratio for the magnetic lines are $3: 4: 1$ as indicated by the results obtained for the magnetizations along any direction in the substrate plane and perpendicular to this plane ${ }^{6-7}$ )

The results of our fit procedure are summarize in table iI.

TABLE II: Hyperfine parameters for the fitted spectra.

\begin{tabular}{|c|c|c|c|}
\hline SAMPLE & $\langle\mathrm{HF}>(\mathrm{KOE})$ & $\mathrm{EQ}(\mathrm{mm} / \mathrm{s})$ & $I(\%)$ \\
\hline 1 & 150 & $-1.0(1)$ & $3.5(1)$ \\
2 & 150 & $-1.0(1)$ & $3.4(1)$ \\
3 & 150 & $-0.9(1)$ & $3.8(1)$ \\
4 & 160 & $-1.1(1)$ & $4.8(1)$ \\
5 & 162 & $-1.1(1)$ & $5.3(1)$ \\
6 & 150 & $-1.3(1)$ & $3.5(1)$ \\
\hline
\end{tabular}

(HF) denotes the hyperfine field at the peak distribution.

EQ and I denote the quadrupolar splitting and intensity for the non magnetic irons

In all cases we obtained quasi-gaussian field distributions, $P(H)$, for the hyperfine fields around the iron atoms (see Fig. 2).

The fact that both the $P(H)$ distibution functions and the average hyperfine fields are very similar for the thin film samples and for the "bulk sample", sample number 6 , allow us to conclude that interfacial irons are not distinguish from the Fe located at the bulk. In all samples the pure paramagnetic doublet shows similar quadrupole interaction and the intensity of this doublet increases as increases the values. This effect can be explained taking into account the increase of the non magnetic $\mathrm{Fe}$ atoms present in the bulk as the modulation length increases.

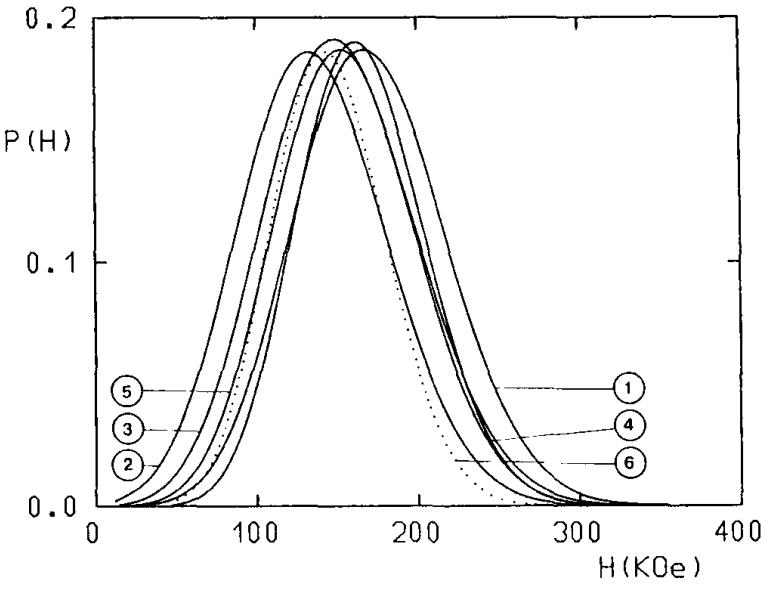

FIGURE 2: Binomial distribution of hyperfine fields.

As we have noticed before, we have carry out magnetic measurements with the aim to determine the behaviour of the masnetization versus the applied magnetic field.

In figure 3 and 4 we show the magnetization dependence on the external field for the easy direction contained in the substrate plane, $M_{\|}$, and in the perpendicular direction to the substrate, $M+9)$.

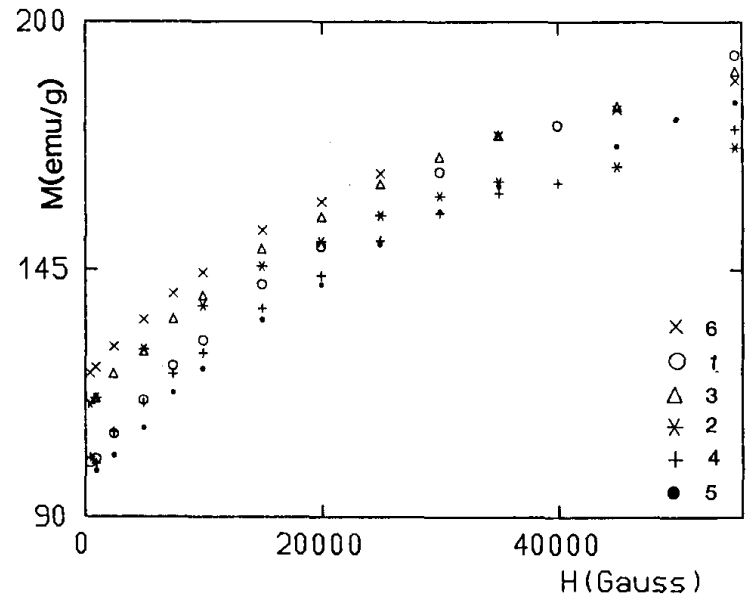

FIGURE 3: $M_{\|}(\mathrm{H})$ curves for the different samples at $T=4.5 \mathrm{~K}$ 


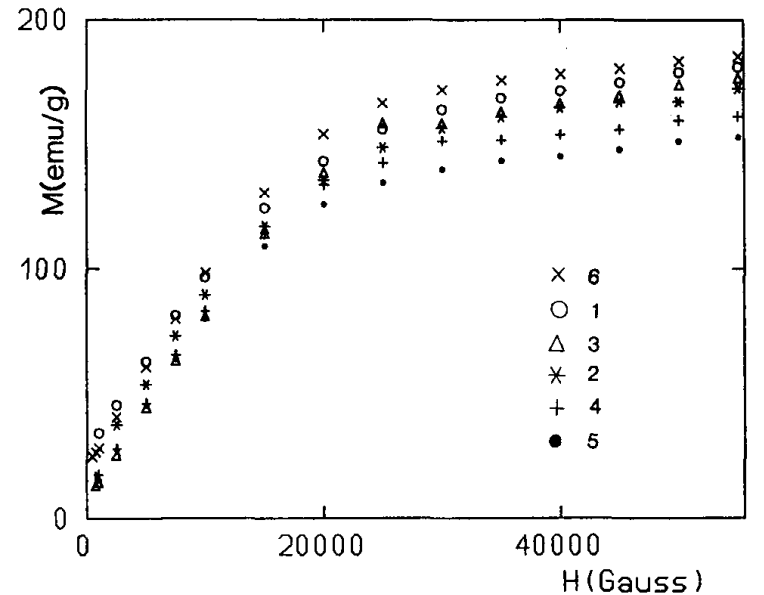

FIGURE 4: $M_{\perp}$ (H) curves for the different samples at $\mathrm{T}=4.5 \mathrm{~K}$

The average dipolar magnetic moment is of about $\left.2 \mu_{B} 10\right)$, where $\mu_{B}$ is the Bohr magneton. At low fields the magnetization along the substrate plane is clearly bigger than in the perpendicular direction. At high fields both magnetizations show the same value, but none of them are saturated existing a nearly linear dependence of $M_{\|}$and $M_{\perp}$ on the applied magnetic fields.

This superimposed susceptibility, $x_{0}$, can be correlated with the speromagnetic ordering of the spins in these thin films as well as with the existence of paramagnetic iron atoms, as has been detected by Mössbauer, which will be thermally agitated giving rise to larger values of $x_{0}$.

The $M \perp$ values decrease with increasing the characteristic modulation lengths. This effect may be attributed to the increase of the number of paramagnetic iron atoms as can be deduced from the Mössbauer spectra which show an increase of the paramagnetic doublet contribution with increasing modulation length 4 ).

\section{REFERENCES}

[1] N.K. Flevaris, J.B. Ketterson and J.E. Hilliard.

J. A.ppl. Phys. 53 (1983) 8046-8051

[2] G. Marchal, P.H. Mangin and Chr. Janot Solid State Commun. 18 (1976) $739-742$

[3] N.K. Jaggi, L.H. Schwartz, H.K. Wong and J.B. Ketterson.

J. Magn. Magn. Mat. 49 (1985) 1-4

[4] B. Martinez, A. Ruiz, A. Labarta, $X$. Obradors, F. Briones and $\mathrm{J}$, Tejada. To be published in IEEE Trans. on Mag.

[5] I. Vincze, Solid State commun. 25 (1978) 689

[6] J. M. Alameda, M. C. Contreras and H. Rubio. Phys. Stat. Sol.(a) $85 \quad(1984) \quad 511$

[7] G. Marchal, Ph. Mangin, M. Piecuch, Chr. Janot and J. Hubsch. J. Phys. F. vol. 7, n.6 (1977)

[8] N. S. Kazama and H. Fujimori Proceedings of the Thin Films Conference. Asilomar, California. U.S.A. (1985)

[9] T. Shinjo, N. Hosoito, K. Kawaguchi, N. Nakayama, T. Takada and Y. Endoh J. Magn. Magn. Mat. 54-57(1986) 737-742

[10] D. Givord, H. S. Li in Nd-Fe permanent magnets. Their present and future applications. Edited by I. V. Mitchell. Brussels and Luxembourg, 1985 